\title{
Toward polymer composites based and architectural engineering induced flexible electrodes for lithium-ion batteries
}

\author{
Jahidul Islam $^{\text {a }}$, Faisal I. Chowdhury ${ }^{\text {a, *, Wahidur Raza }}{ }^{\text {a }}$, Xianghui Qi ${ }^{\text {b }}$, M. Rezaur Rahman ${ }^{\text {c }}$ \\ Jagotamoy Das ${ }^{\mathrm{d}}$, Jamal Uddin ${ }^{\mathrm{e}}$, Hossain M. Zabed ${ }^{\mathrm{b}, * *}$ \\ ${ }^{a}$ Department of Chemistry, University of Chittagong, Chittagong, 4331, Bangladesh \\ ${ }^{\mathrm{b}}$ School of Food and Biological Engineering, Jiangsu University, Zhenjiang, 212013, Jiangsu, China \\ ${ }^{c}$ Department of Chemical Engineering and Energy Sustainability, Faculty of Engineering, Universiti Malaysia Sarawak (UNIMAS), 94300, Kota Samarahan, Sarawak, \\ Malaysia \\ d Department of Pharmaceutical Sciences, Leslie Dan Faculty of Pharmacy, University of Toronto, College St., ON, M5S 3M2, 910-144, Canada \\ ${ }^{\mathrm{e}}$ Center for Nanotechnology, Department of Natural Sciences, Coppin State University, Maryland, USA
}

\section{A R T I C L E I N F O}

\section{Keywords:}

Polymer composites

Architectural engineering

Customized electrodes

Flexible electrodes

Lithium-ion batteries

Energy storage

\begin{abstract}
A B S T R A C T
Recently, polymers, especially conducting (CPs) and non-conducting polymers (nCPs), have been emerged as the promising flexible electrode components for lithium-ion batteries due to their inherent high mechanical tolerance limit, excellent thermal and chemical stability, low density, ease of processing, low cost, and versatility. In addition, CPs provide good electrical conductivity. Polymeric structures remain almost the same even after hundreds to thousands of electrochemical cycles. However, some crucial factors, such as low conductivity, energy density, and rate performance, often limit the large-scale exploitation of these polymers. Although CPs, and nCPs can provide the desired flexibility, nCPs, in particular, increase the 'dead volume' of electrodes. In this context, it is necessary to resolve the issues existing with the polymers to make them effective confinement matrices for flexible electrodes. On the other hand, customizing the electrode architectures is vital for achieving multidirectional flexibility without compromising energy density and overall capacity. However, low active materials loading and deviation from the customized structures after several deformation cycles still affect the desired performance in terms of electrochemical and mechanical. Furthermore, the intricate and costly preparation processes of customized electrodes are the major bottlenecks toward practical applications. This review discusses the recent progress, merits, and demerits of the most widely studied polymer composites-based and architectural engineering induced flexible electrodes for lithium-ion batteries (LIBs). Both CPs and nCPs are discussed in the perspectives of current research status, major limitations, key factors associated with electrochemical performances and future outlook of the developments on polymer-based flexible electrodes.
\end{abstract}

Abbreviations: AQ, 2,6-diaminoanthraquinone; CPs, conducting polymers; CMC, carboxymethyl cellulose; CAs, conductive additives; CNs, cellulose nanomaterials;

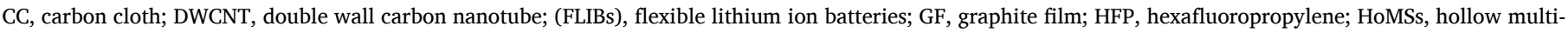

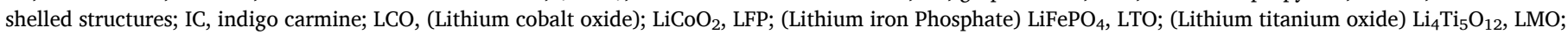

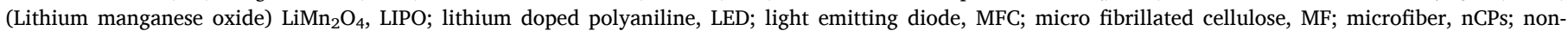

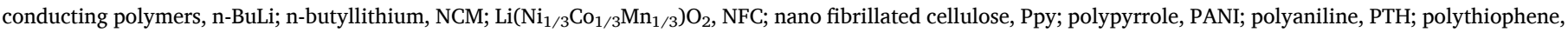

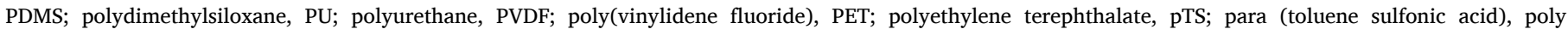

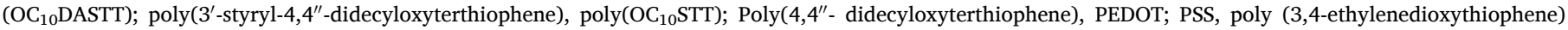

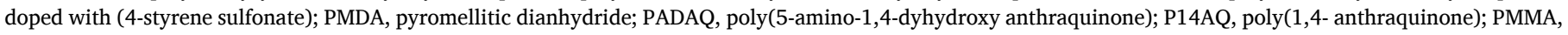

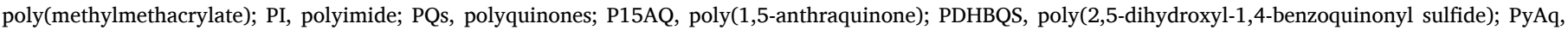

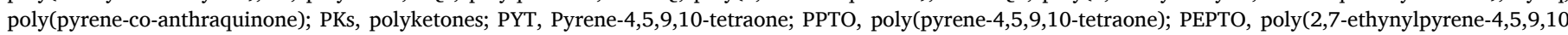

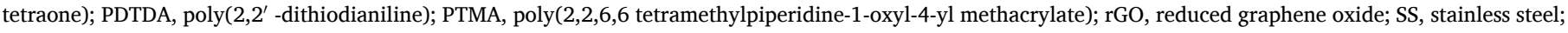

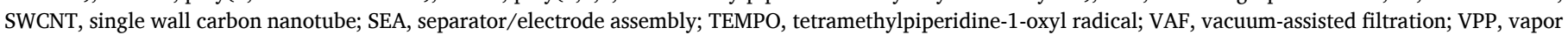
phase polymerization.

* Corresponding author.

$* *$ Corresponding author.

E-mail addresses: faisal@cu.ac.bd (F.I. Chowdhury), zabed@ujs.edu.cn, zabedctgbd@yahoo.com (H.M. Zabed). 